\title{
A Contextual Classifier That Only Requires One Prototype Pixel for Each Class
}

\author{
Gabriela Maletti, Bjarne Ersbøll, and Knut Conradsen
}

\begin{abstract}
A three-stage scheme for the classification of multispectral images is proposed. In each stage, statistics of each class present in the image are estimated. The user is required to provide only one prototype pixel for each class to be seeded into a homogeneous region. The algorithm starts by generating optimum initial training sets, one for each class, maximizing the redundancy in the data sets. These sets are the realizations of the maximal discs centered on the prototype pixels for which it is true that all the elements belong to the same class as the center one. Afterwards, a region-growing algorithm increases the sample size, providing more statistically valid samples of the classes. Final classification of each pixel is done by comparison of the statistical behavior of the neighborhood of each pixel with the statistical behavior of the classes. A critical sample size obtained from a model constructed with experimental data is used in this stage. The algorithm was tested with the Kappa coefficient $\kappa$ on synthetical images and compared with $K$-means $(\bar{\kappa}=0.41)$ and a similar scheme that uses spectral means $(\bar{\kappa}=0.75)$ instead of histograms $(\bar{\kappa}=0.90)$. The results are shown on a dermatological image with a malignant melanoma.
\end{abstract}

Index Terms-Prototypes, redundancy, region growing, supervised classification, window size optimization.

\section{INTRODUCTION}

A PREMISE to a supervised classifier is that the training sets provided are statistically valid samples of the classes. Many semi-automatic training and validation set generation schemes by means of region-growing algorithms [1], [2] have been proposed. However, these schemes still require some user-input for the estimation of the parameters of the classes. It could be an advantage to develop schemes that minimize the amount of information the user is required to provide. On the other hand, contextual classifiers produce higher classification accuracy. However, the definition of an optimum window size for each step of the image analysis is still a problem [4].

From a semantic and pragmatic point of view [5], a window defines a subset of an entity in which the instances are spatially connected. From a heuristic point of view, the initial optimum window for a given prototype pixel defines the realization of the maximal disc centered on it for which it is true that all the elements belong to the same class as the center one. This can be obtained by detecting the emergence of a state of higher order through the minimization of an optimal learning curve that depends on the fraction of examples [6]. Based on the Heisen-

Manuscript received November 23, 2001; revised March 22, 2002. This work was supported by the SITE Project funded by a grant from the Danish Technical Research Foundation (Project Number STVF 56-00-0123).

The authors are with the Section for Image Analysis and Computer Graphics, Informatics and Mathematical Modeling (IMM), Technical University of Denmark (DTU), 2800-DK Kgs. Lyngby, Denmark (e-mail: gmm@imm.dtu.dk; be@imm.dtu.dk; kc@imm.dtu.dk).

Publisher Item Identifier S 0018-9499(02)06167-1. berg Uncertainty Principle, it can be deduced that this optimal learning curve corresponds to cases for which the envelope of the signal or the signal itself is a Gaussian function. From the Central Limit Theorem, we know that the convolution of a large number of positive functions is approximately a Gaussian function. Since consecutive values of the function resulting from convolution of two other functions contain a high degree of redundancy [7], we choose as trajectory for the learning curve the points for which the redundancy in the envelope of the fraction of examples is maximized. In the present work, the estimated optimum size of the neighborhood of each prototype pixel is obtained at the minimum of an energy function computed for the most redundant set that can be generated for each window size. This stage will be called "the initial training set generation scheme" [8].

A statistically valid sample of the class can then be obtained by increasing the sample size by means of a "region growing" algorithm [9]. Once the samples are defined, the minimum amount of information needed for classifying each pixel in the image has to be established. This is done based on the relationship between the window size and separability between classes obtained from experimental data, which provides a way of computing the critical number of examples needed for inferring an underlying structure in the data. Pixels are finally classified by comparison of the statistical behavior of its neighborhood with the statistical behavior of the classes [2].

\section{RATIONALE OF THE ALGORITHM}

Let a specific image with $B$ noncorrelated bands $X=\{x[r, c]\}$ defined over the given domain $L=\{[r, c] \mid 1 \leq$ $r \leq M, 1 \leq c \leq N\}$ with $Q$ quantization levels. Let $K$ be the number of prototype pixels $x_{k}^{0} \in X, 1 \leq k \leq K$, seeded into homogeneous regions.

From a syntactic point of view [5], let a window $D_{w}[r, c]$ define the realization of a disc $\mathrm{D}_{w}[r, c]$ of radius $w$ centered on the position $[r, c]$ of a given pixel

$$
\begin{array}{r}
D_{w}[r, c]=\left\{d_{w}[p]=x[r-i, c-j] \mid 0 \leq\right. \\
\left.i^{2}+j^{2} \leq w^{2} \wedge 1 \leq p \leq n_{w}\right\}
\end{array}
$$

where $n_{w}$ is the number of pixels belonging to the set $D_{w}[r, c]$.

\section{A. The Initial Training Set Generation Scheme}

For each prototype pixel $x_{k}^{0}$, an active learning process with increasing window size for each iteration is defined (see the left side of Fig. 1). It is expected that, at some point, the class boundary is detected. This point will be associated with the optimum window size $n_{t o}$ for the given pixel. For each window 


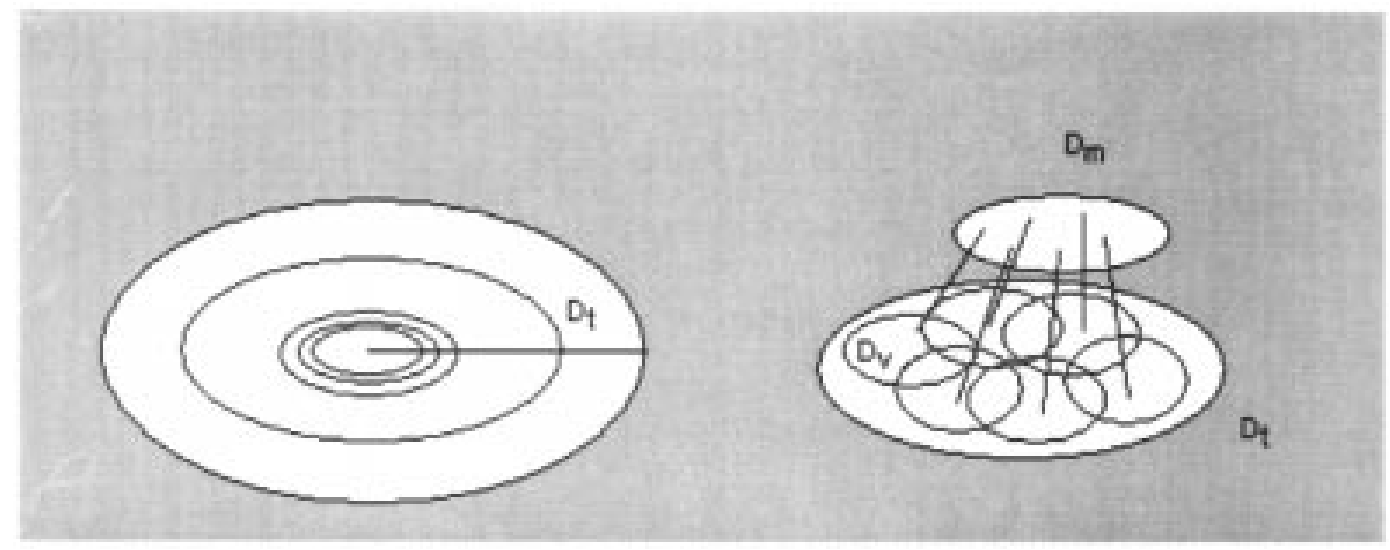

Fig. 1. The mapping model. To the left, a set of discs of increasing radius $t$ centered on a given pixel is shown. To the right, one of these discs is shown in detail: overlapping discs of a fixed radius $v$ are placed into the disc of radius $t$. A new disc of radius $m$ is constructed with the centers of these overlapping discs of radius $v$. The mapping model is completely defined when weights are assigned to the elements of each disc.

size, the means of the realizations of all the discs of the same size that can be placed into this window are estimated. The estimated variance of the estimated means within groups is then tracked and minimized over the line of maximum redundancy of the mapping model [8].

In general, let the estimation $y$ of the mean of a realization $D_{w}$ of a disc $\mathrm{D}_{w}$ be the projection ${ }^{1}$ of the realization $D_{w}$ over the weighting function $\omega_{w}$

$$
y_{w}=\sum_{p} d_{w}[p] \omega_{w}[p]
$$

where $d_{w}[p]$ is the $p$ th pixel belonging to $D_{w}$ and $\omega_{w}[p]$ is the weight assigned to it.

Let the realization of a disc $\mathrm{D}_{m, v}[r, c]$ be composed by the estimations $y_{v}[r-i, c-j] \mid 0 \leq i^{2}+j^{2} \leq m^{2}$ of the means within $n_{m}$ groups $D_{v}[r-i, c-j]$ of size $n_{v}$ using the same set of weights $\omega_{v}$. Let $y_{m, v}[r-i, c-j]$ be the estimated mean between these groups using the set of weights $\omega_{m}$.

Since, for all realization $D_{v, m} \exists D_{t} \wedge \omega_{t}$ such that $t=t(v, m) \simeq v+m$ and $\omega_{t} \simeq \omega_{v} * \omega_{m},{ }^{2}$ the redundancy of a given pair $t(v, m)$ is defined as

$$
R\left(\mathrm{D}_{t}\right)=1-\frac{S\left(\mathrm{D}_{t}\right)}{\log n_{t}}
$$

where $n_{t}$ is the number of pixels in the disc $\mathrm{D}_{t}$ and $S\left(\mathrm{D}_{t}\right)$ is the entropy of the disc defined as

$$
S\left(\mathrm{D}_{t}\right)=-\sum_{p=1}^{n_{t}} \omega_{t}[p] \log \left(\omega_{t}[p]\right)
$$

where $n_{t}$ is the number of pixels of the disc, and $0<\omega_{t}[p]<1$ is the weight assigned to the $p$ th pixel of the disc.

As can be observed, the measure of redundancy used depends on the group size $n_{v}$ and the number of groups $n_{m}$, and it is inversely related to the entropy of the weights $\omega_{v}$ and $\omega_{m}$ assigned to the elements of the groups and to the groups, respectively.

\footnotetext{
${ }^{1}$ Scalar product.

${ }^{2}$ The equality holds for squares but small differences can be obtained in the case of discs.
}

For a given prototype pixel $x_{k}^{0}$ with position $[a, b]$, the size of the initial training set $n_{t o}$ corresponds with the minimum estimated variance of the estimated means within groups. This function is computed for each window size for the most redundant set

$$
\mathrm{Z}^{2}\left[t\left(v_{o}, m_{o}\right)\right]=\sum_{p=1}^{n_{m_{o}}} \omega_{m_{o}}\left\|y_{v_{o}}[p]-y_{v_{o}, m_{o}}[a, b]\right\|^{2}
$$

where $\left(v_{o}, m_{o}\right)$ is the pair for which the redundancy is maximal for each fixed radius $t(v, m),\|\|$ is the Euclidian norm, $y_{v_{o}}[p] \in D_{m_{o}, v_{o}}[a, b]$ are the estimated means within groups, and $y_{v_{o}, m_{o}}[a, b]$ is the estimated mean between groups. The radius $t$ is increased in such a way that the internal-to-external entropy ratio [8] is smaller than or equal to a constant $R_{0} \forall t$ used. Then

$$
t_{o}=\min \left(Z^{2}\left[t\left(v_{o}, m_{o}\right)\right]\right) \text {. }
$$

Note that, in order to simplify the notation, a small change will be made. Hereafter the notations $v$ and $m$ are substituted for the notations $v_{o}$ and $m_{o}$ of the optimum radius $t_{o}$.

\section{B. The Region-Growing Algorithm}

Once the initial training sets are defined, the sample size can be increased by means of an optimized region-growing algorithm. Following the approach originally proposed in [2], [9], and [10], for each class, pixels satisfying a homogeneity criterion are included in the grown region. This criterion is evaluated in a window of optimum size $n_{v}$ obtained in the Initial Training Set Generation Stage. A pixel is aggregated into the region if the difference between the homogeneity value of the class and the homogeneity value of the pixel centered window does not exceed a certain threshold. The growing of a region stops, once the homogeneity criterion is no longer satisfied. Following the notation previously introduced, let $G_{0}=\left\{x^{0}[a, b]\right\}$ be the initial subregion pointing out a class. In general, $\forall g>0$ let $G_{g-1}^{\prime}$ be the set of pixels that do not belong to $G_{g-1}$ but having at least a neighbor with $G_{g-1}$ under a certain connectivity. The set $G_{g}$ is the region jointly formed by $G_{g-1}$ and the pixels of $G_{g-1}^{\prime}$ such that the distance from the estimated mean of the class and the 


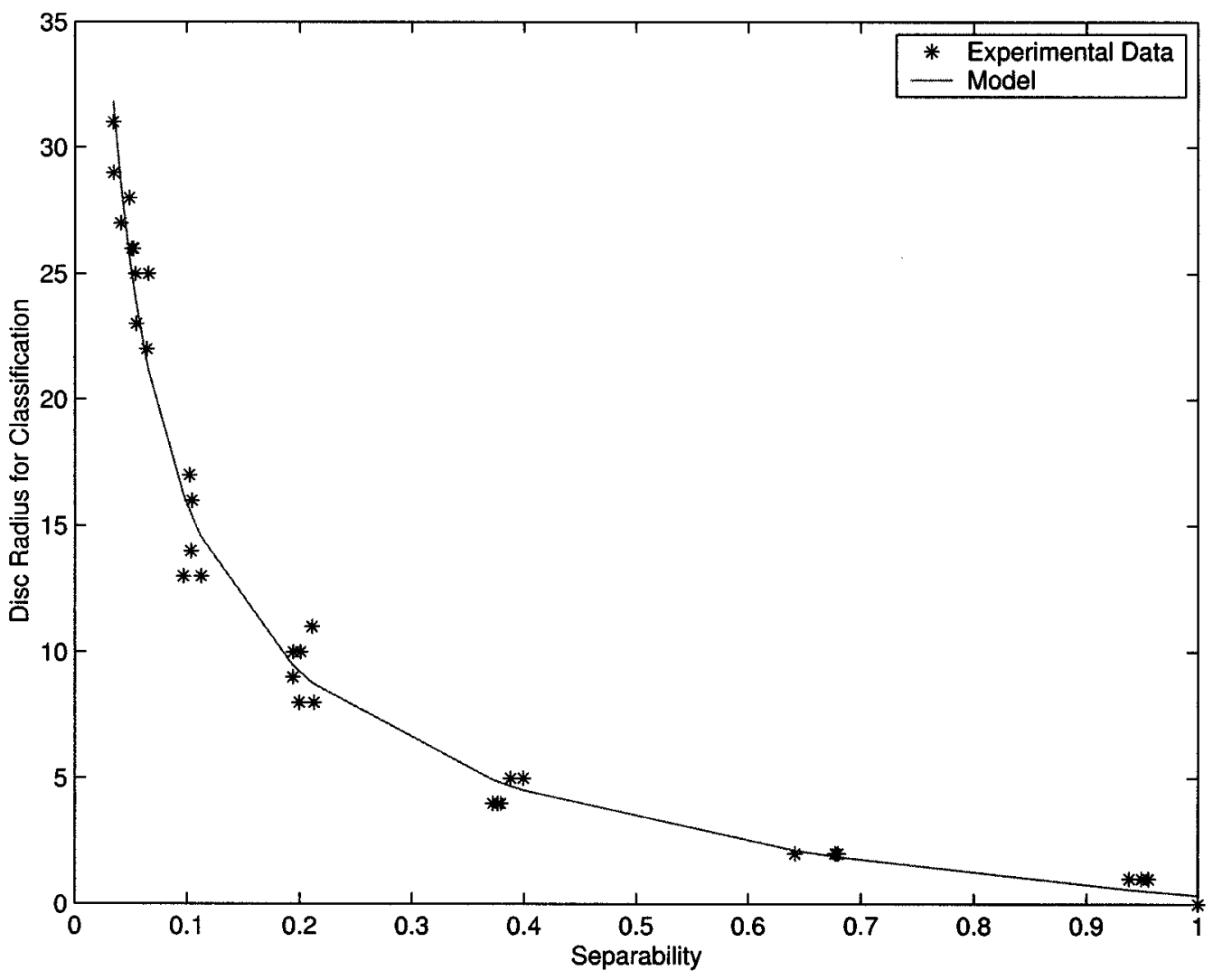

Fig. 2. Separability $d A$ versus optimum disc radius $w_{c}$ for classification.

estimated mean of the neighborhood centered on those pixels does not differ more than a certain threshold

$$
G_{g} \equiv\left\{x[q, r]^{g}:\left\|y_{v}[q, r]-y_{m, v}[a, b]\right\| \leq \beta Z\left[t_{o}\right]\right\}
$$

where \|\| is the Euclidian norm and $\beta \in R^{+}$. The growing of a region continues until $G_{l+1}=G_{l}$.

\section{The Final Classification}

In this last stage, the normalized histograms of the grown regions of each class are used. An optimum window size for image classification is defined using these histograms. Afterwards, the following measure of distance from each pixel to each class is computed; it is the pondered sum of the difference of areas of pairs of class density functions:

$$
d A_{(i),(j)}=\frac{1}{2 B} \sum_{b=1}^{B} \sum_{n=1}^{Q}\left|h_{(i)}[b, n]-h_{(j)}[b, n]\right|
$$

where $B$ is the number of bands of the image, $Q$ is the number of quantization levels, and $h_{(i)}$ and $h_{(j)}$ are the per band normalized histograms of the $i$ th and $j$ th class, respectively.

The critical number of samples for classification is computed as a function of the minimal separability between neighbor classes. This empirical model was constructed doing linear regression of experimental data (see Appendix A)

$$
w_{c}=-6.8341+\frac{7.1800}{\sqrt{d A}}
$$

where $d A$ has the same meaning as before and $w_{c}$ is the radius of the estimated optimum disc for classification. It is optimum in the sense that it corresponds to the best classification rate for a set of experimental data with similar statistical descriptions. Fig. 2 shows the graphical behavior of this relation.

A pixel $x[s, t]$ belonging to the multispectral image $X$ is classified according to the following criterion:

$$
x[s, t] \rightarrow k: d A_{(x),(k)}, \text { minimum, } \forall k
$$

where $_{(k)}$ represents the normalized histogram $h_{(k)}$ of the grown region corresponding to the $k$ th class and $(x)$ represents the normalized histogram $h_{(x)}$ of the neighborhood of radius $w_{c}$ centered on the position $[s, t]$ of the pixel $x$.

\section{RESULTS AND DISCUSSION}

In order to evaluate the algorithm, a set of 15 synthetic images with signal-to-noise ratios (SNR) ${ }^{3}$ SNR of $0,3,8,15$ and 26 and number $O$ (from order) of classes of 3, 5, and 7 was generated. The separation between consecutive means of the classes was five gray levels for all images. The classes are Gauss-distributed. The size of the images is $128^{2}$ pixels (see Fig. 3). The prototype pixels were seeded in suitable places. The initial optimum window size was estimated for each prototype pixel of each synthetic image setting the internal-to-external-entropy ratio $R_{o}$ [8] to an empirically found value of 10 . The weight assigned to each pixel in each disc was the inverse of the size of the respective

\footnotetext{
${ }^{3}$ The SNR is defined as SNR $=-20[(K-1) \sigma]^{-1} \log \sum_{i=1}^{K-1}\left|\mu_{i}-\mu_{i+1}\right|$ where the $\mu_{i}$ is the mean of the $i$ th class, the means are ordered in increasing size, and $\sigma$ is the standard deviation of each one of the $K$ classes.
} 

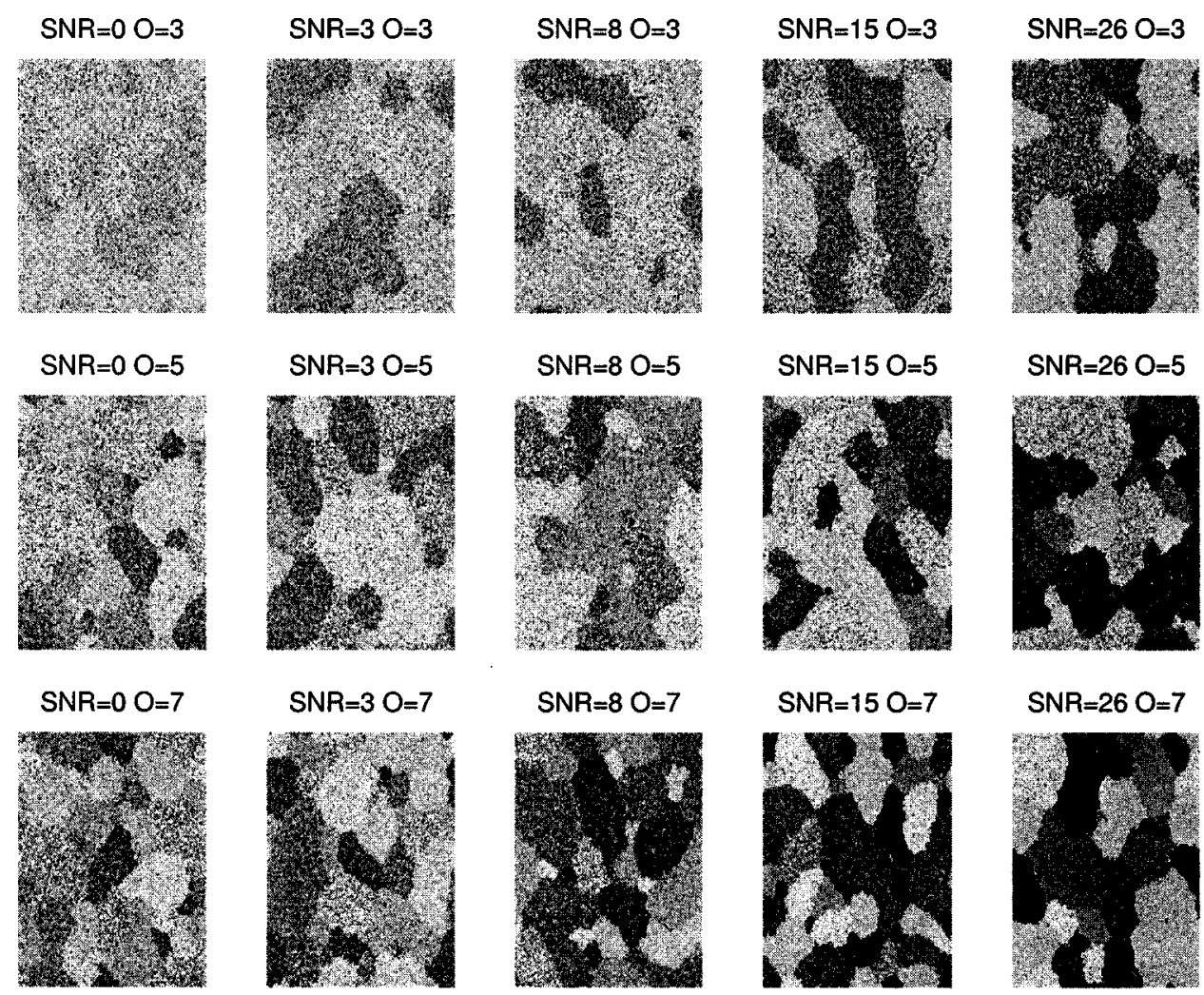

Fig. 3. The original set of synthetical images.
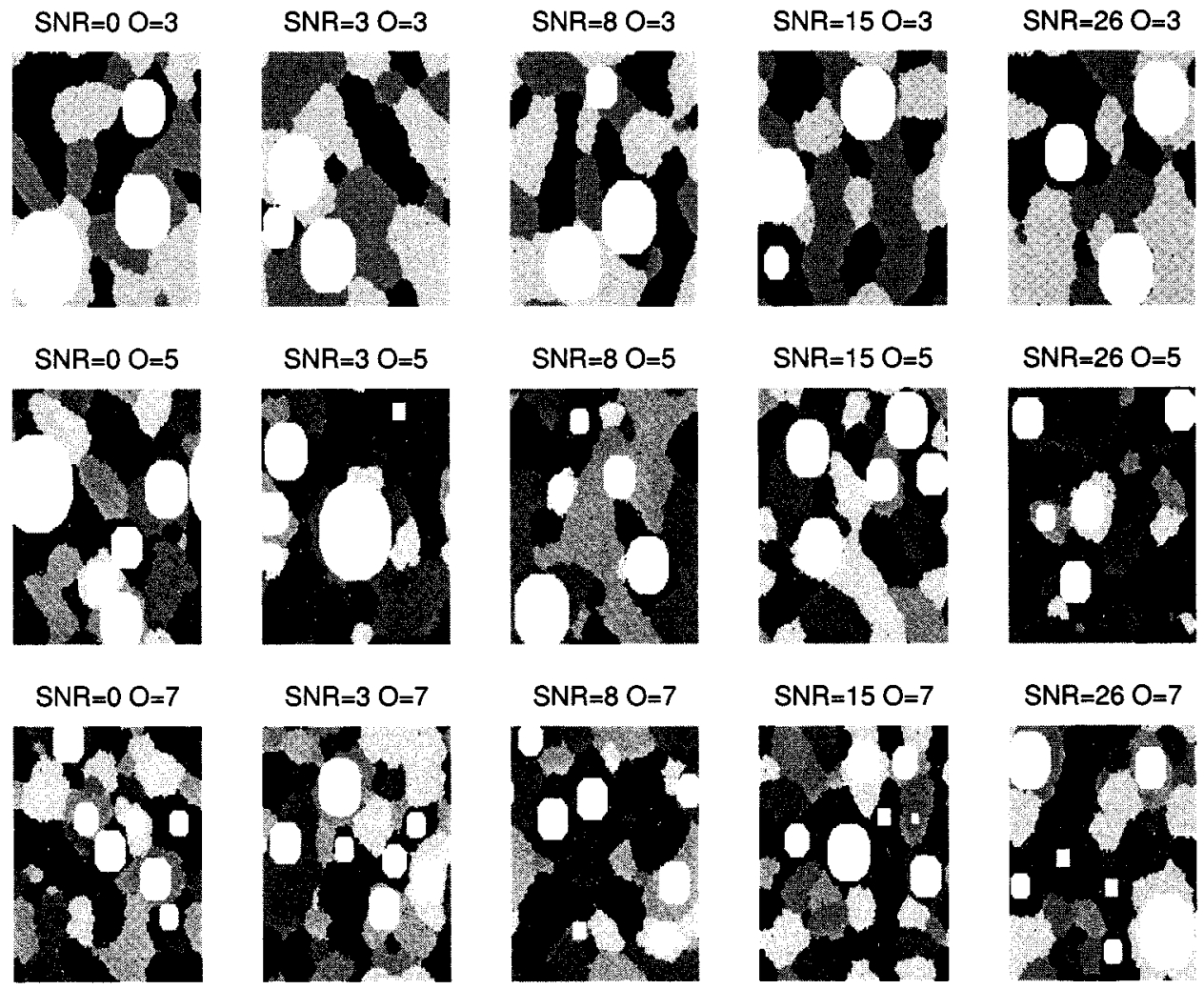

Fig. 4. The initial training sets (in white) centered on the seeded prototype pixels overlayed with the real thematic maps of the synthetical images shown in Fig. 3.

disc for all the examples treated. The initial training sets generated are shown in Fig. 4. The outputs of the second and third stage of the present scheme are presented in Figs. 5 and 6, respectively. 

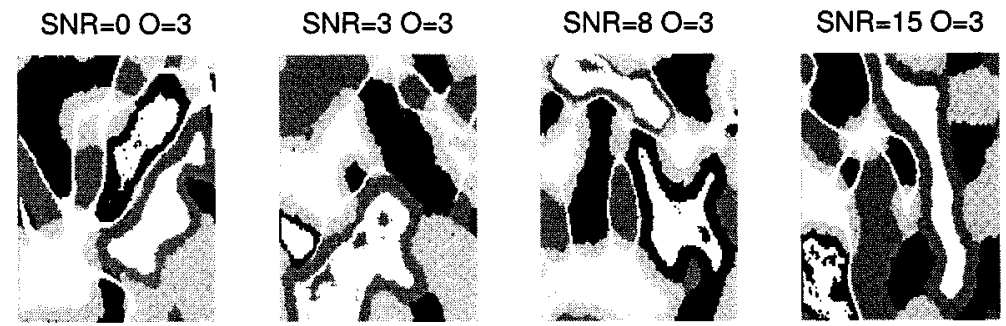

$S N R=26 \quad O=3$

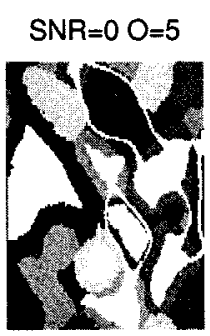

$\mathrm{SNR}=3 \mathrm{O}=5$

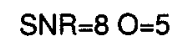

$\mathrm{SNR}=15 \mathrm{O}=5$
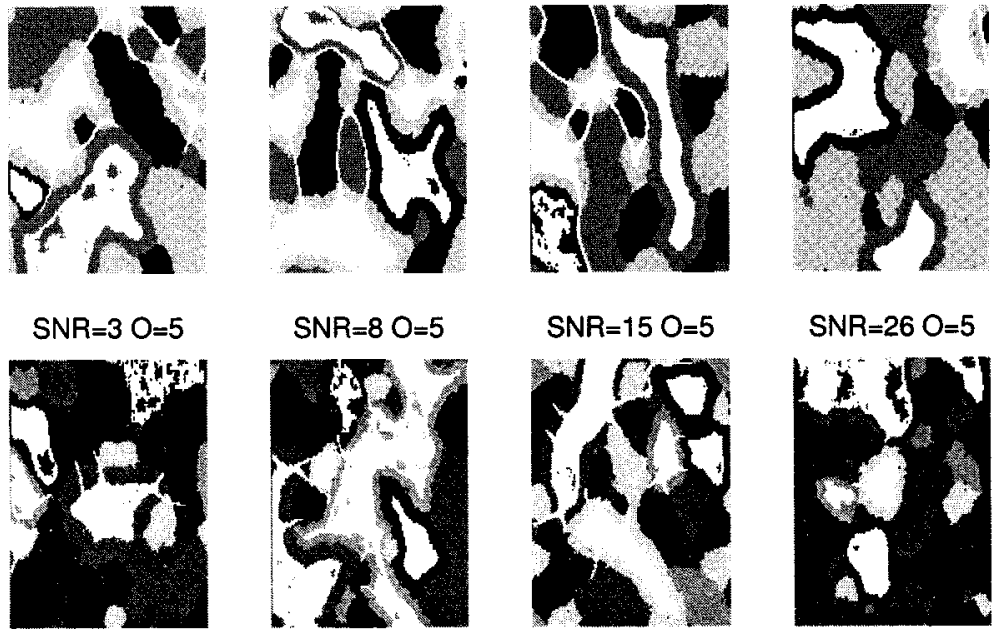

$S N R=26 \quad O=5$

$\mathrm{SNR}=0 \quad \mathrm{O}=7$

$\mathrm{SNR}=3 \mathrm{O}=7$

$\mathrm{SNR}=8 \mathrm{O}=7$

SNR $=15 \mathrm{O}=7$
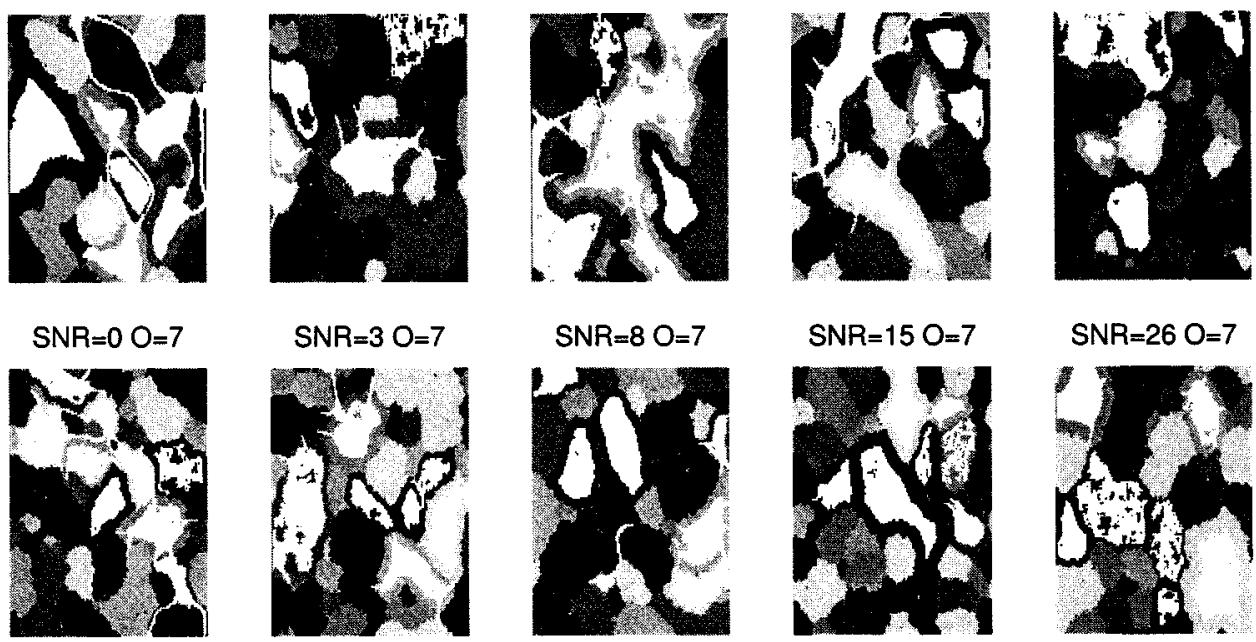

$\mathrm{SNR}=26 \mathrm{O}=7$

Fig. 5. The grown regions (in white) generated using the initial training sets delineated in Fig. 4 overlayed with the real thematic maps of the synthetical images shown in Fig. 3.
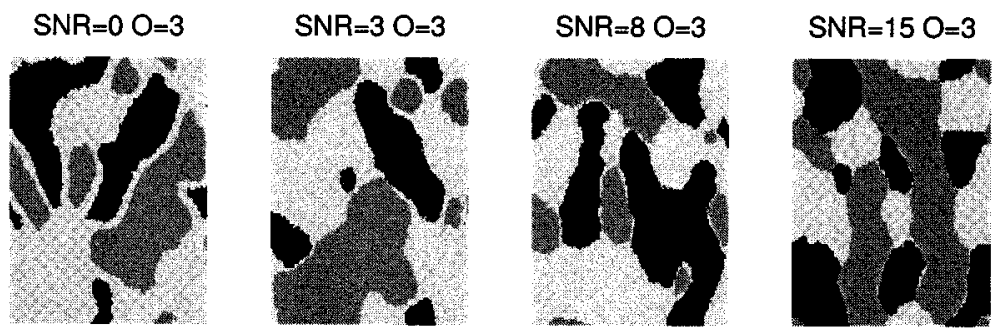

$\mathrm{SNR}=26 \mathrm{O}=3$

$\mathrm{SNR}=0 \mathrm{O}=5$

$\mathrm{SNR}=3 \mathrm{O}=5$

$S N R=8 \quad O=5$

$\mathrm{SNR}=15 \mathrm{O}=5$
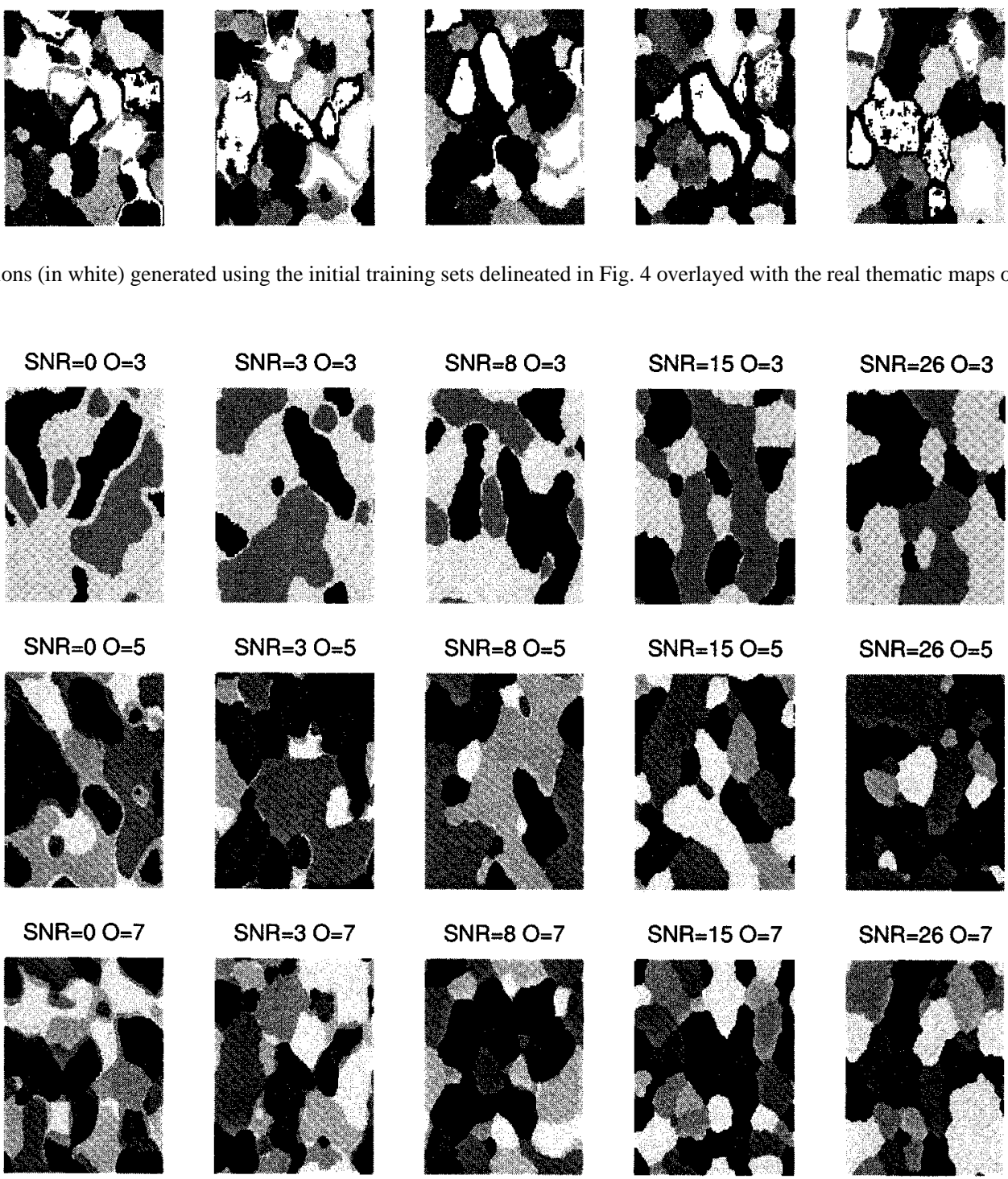

SNR $=26 \quad 0=7$

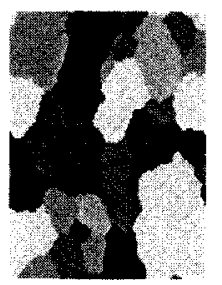

Fig. 6. The estimated thematic maps generated by the present classifier for the set of synthetical images shown in Fig. 3 using the training sets delineated in Fig. 5 .

The output of the algorithm for this set of synthetical images was tested with Cohen's Kappa coefficient $\kappa$. It was compared to $K$-means and a scheme similar to the present one, except that in the final classification stage the spectral means of each pixel 
TABLE I

KAPPA VALUES OF THE DiFFERENT ClassificATION OUTPUTS

\begin{tabular}{cccccc}
\hline SNR & 0 & 3 & 8 & 15 & 26 \\
\hline \hline \multicolumn{5}{c}{ Order $O=3$} \\
\hline \hline K-Means & 0.0352 & 0.0565 & 0.0911 & 0.7206 & 0.9295 \\
Spectral Means & 0.6991 & 0.8718 & 0.8692 & 0.8902 & 0.9565 \\
Norm. Histograms & 0.7967 & 0.9021 & 0.9164 & 0.9498 & 0.9799 \\
\hline \hline \multicolumn{5}{c}{ Order $O=5$} \\
\hline \hline K-Means & 0.1186 & 0.1188 & 0.3172 & 0.6964 & 0.9395 \\
Spectral Means & 0.5484 & 0.5925 & 0.7812 & 0.8281 & 0.8672 \\
Norm. Histograms & 0.7350 & 0.8884 & 0.9414 & 0.9583 & 0.9738 \\
\hline \hline \multicolumn{6}{c}{ Order $O=7$} \\
\hline \hline K-Means & 0.1716 & 0.2537 & 0.2592 & 0.4766 & 0.9347 \\
Spectral Means & 0.3995 & 0.6312 & 0.6465 & 0.7225 & 0.8741 \\
Norm. Histograms & 0.7211 & 0.8511 & 0.9204 & 0.9543 & 0.9749 \\
\hline
\end{tabular}

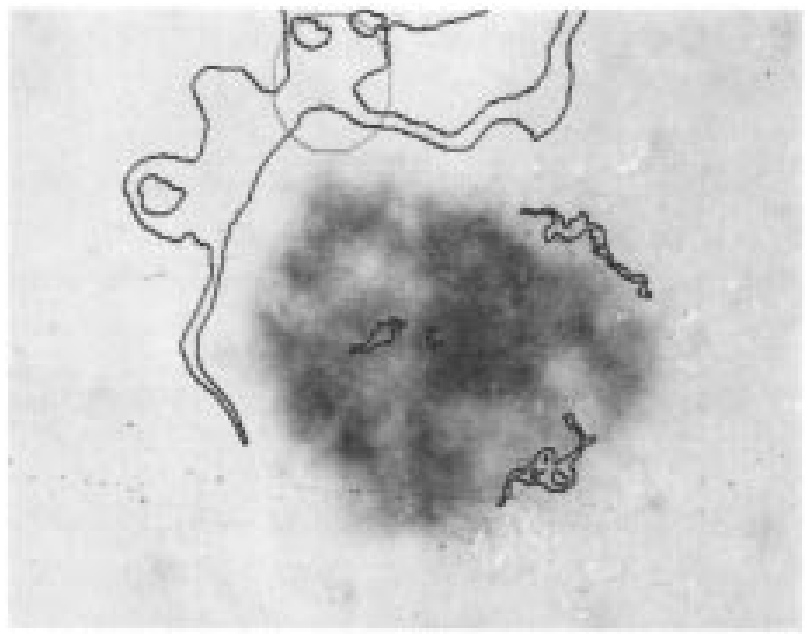

Fig. 7. The initial training sets (delineated with circles) centered on the seeded prototype pixels overlayed with the grown regions (delineated in black) of the image of a malignant melanoma.

centered neighborhood are computed and the distance between these means and the estimated means of the classes are used for assigning the pixels to the classes (see Table I for details).

Testing the algorithm for dermatological examples introduced some practical considerations that follow. First, the main problem in dermatological images is precisely defining all the classes and their homogeneity. The seed of the prototype pixels is crucial in the sense that this will set the parameters for the region-growing algorithm. Too homogeneously grown regions will produce classes with almost total separability and, therefore, the final classification will be done with too small window sizes not defining representative real large variation neighborhoods. On the other hand, if the pixels are seeded into regions with large heterogeneity, a high overlap in the statistics of the grown regions can occur, and the final classification stage will have to handle with large neighborhoods in order to make a good discrimination, but border effects can be introduced.

Secondly, since all the pixels are classified, thematic maps produced for real examples can contain misclassified pixels (healthy skin classified as ill and vice versa). This can be considered as a kind of noise that can be reduced by applying an iterative median filter to the thematic map.

An image of a malignant melanoma is the real case presented in this paper (see Fig. 7). The size of the original image was

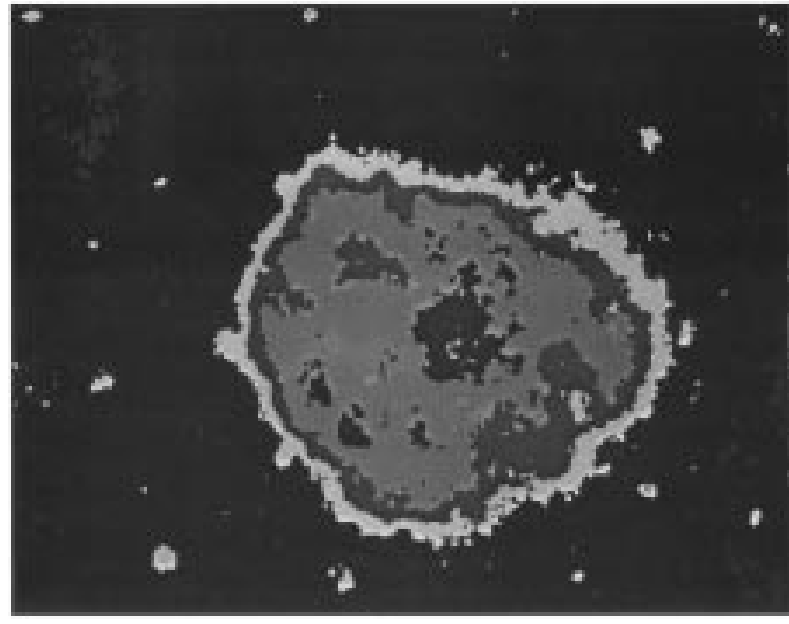

Fig. 8. The thematic map produced by the algorithm for the image.

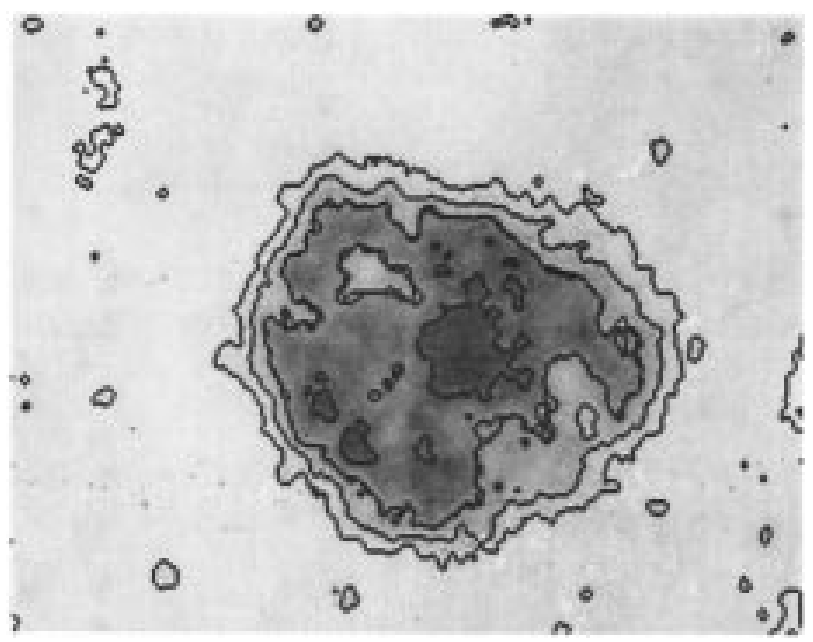

Fig. 9. The original image overlayed with the thematic map after three iterations of the median filter.

885 by 590 pixels. The eigenvalues of the principal components were in descending order: $0.209849,0.001498$, and 0.000137 . The two principal components of the original image reduced $50 \%$ in size were used as input to the algorithm. Five prototype pixels pointing each one to a class were seeded into homogeneous regions. The scheme produced a thematic map containing misclassified pixels (see Fig. 8), the number of which was reduced by applying an iterative median filter to the thematic map (see the overlay with the original image reduced by $50 \%$ in Fig. 9). Pixels with no overlap to one of the classes were assigned to a reject class.

\section{CONCLUSION}

A new contextual classifier for multispectral images has been developed. The scheme has been tested for synthetic and real examples. It has been shown that the application of the iterative median filter to the thematic map can improve the quality of the results in real examples. In general, results are satisfying with respect to both numerical evaluation and visual appreciation. 
TABLE II

Distance $d A$ Between Pairs of Classes Given by the SEParation BETWEen THEIR MEANS $s \mu$ AND THEIR STANDARD DEVIATION $\sigma$

\begin{tabular}{ccccccc}
\hline$s \mu / \sigma$ & 1 & 2 & 4 & 8 & 16 & 32 \\
\hline 0.5 & 0.2130 & 0.1035 & 0.0511 & 0.0349 & 0.0345 & 0.0549 \\
1 & 0.3994 & 0.1998 & 0.0968 & 0.0488 & 0.0411 & 0.0543 \\
2 & 0.6416 & 0.3720 & 0.1946 & 0.1022 & 0.0659 & 0.0523 \\
4 & 0.9383 & 0.6797 & 0.3879 & 0.1938 & 0.1046 & 0.0644 \\
8 & 0.9999 & 0.9493 & 0.6772 & 0.3767 & 0.2111 & 0.1127 \\
16 & 1.0000 & 1.0000 & 0.9555 & 0.6797 & 0.3790 & 0.2010 \\
\hline
\end{tabular}

TABLE III

Disc RAdius $w_{c}$ CORRESPONDING TO THE Best KAPPA VALUe (ONE) FOR Each Pair of Classes Given by the SEParation BetweEn Their MEANS $s \mu$ AND THEIR STANDARD DEVIATION $\sigma$

\begin{tabular}{ccccccc}
\hline$s \mu / \sigma$ & 1 & 2 & 4 & 8 & 16 & 32 \\
\hline 0.5 & 8 & 14 & 26 & 29 & 31 & 23 \\
1 & 5 & 8 & 13 & 28 & 27 & 25 \\
2 & 2 & 4 & 10 & 17 & 25 & 26 \\
4 & 1 & 2 & 5 & 9 & 16 & 22 \\
8 & 0 & 1 & 2 & 4 & 11 & 13 \\
16 & 0 & 0 & 1 & 2 & 4 & 10 \\
\hline
\end{tabular}

\section{APPENDIX A}

\section{A. The Empirical Relation Window Size for Classification-Separability Between Classes}

A set of 36 pairs $(A, B)$ of synthetic images $128^{2}$ pixels in size was generated. The values of the pixels were generated using different Gauss probability density functions $N\left(\mu_{A}, \sigma_{A}^{2}\right)$ and $N\left(\mu_{B}, \sigma_{B}^{2}\right)$. The values of the parameters were $\sigma_{A}=\sigma_{B}=$ $2^{j-1}, j=1 \ldots 6$ and $\mu_{A}=128+\left(\left(2^{(i-1)}\right) / 2\right)$ and $\mu_{B}=$ $\mu_{A}+\left(\left(2^{(i-1)}\right) / 2\right), i=1 \ldots 6$.

For each pair of images $(A, B)$, neighborhoods (discs) of increasing size were centered on the pixels from rows 33 to 96 and columns 33 to 96 of each one of the images. The radius of the discs varied from 0 to 31 .

For each window size:

- the normalized histogram of each pixel centered neighborhood was computed;

- the distance $d A$ from this histogram to the normalized histogram of both classes was calculated [see (8)];

- the pixel was assigned to the closest class;
- the quality of the final classification was measured with Cohen's Kappa coefficient $\kappa$.

The optimum radius for each pair of images corresponded to the maximum Kappa value of one.

Tables II-III show the distances between each considered pairs of classes and the corresponding optimum disc radii for classification.

\section{ACKNOWLEDGMENT}

The authors would like to thank the National Hospital of Denmark for providing the digital images used and J. L. Chávez from the Laboratory of Remote Sensing of the Institute for Geophysics of the National Autonomous University of Mexico for providing source code of the package SANDI (Digital Image Analysis System) used in the development of the present scheme.

\section{REFERENCES}

[1] H. Flesche, A. A. Nielsen, and R. Larsen, "Supervised mineral classification with semi-automatic training and validation set generation in scanning electron microscope energy dispersive spectroscopy images of thin sections," Math. Geol., vol. 32, no. 3, pp. 333-366, 2000.

[2] J. Lira and G. Maletti, "A supervised classifier for multispectral and textured images based on an automated region growing algorithm," Eur. Space Agency Publ., vol. SP-434, pp. 153-158, 1998.

[3] J. Lira and L. Frulla, "An automated region growing algorithm for segmentation of texture regions in SAR images," Int. J. Remote Sensing, vol. 19, no. 18, pp. 3595-3606, 1998.

[4] M. Hodgson, "What size window for image classification? a cognitive perspective," Photogramm. Eng. Remote Sensing, vol. 64, no. 8, pp. 797-807, 1998.

[5] J. Sowa, "Peircean fundations for a theory of context," Lecture Notes Comput. Sci., vol. 1257, pp. 41-64, 1997.

[6] A. Buhot and M. Gordon, "Phase transitions in optimal unsupervised learning," Phys. Rev. E, vol. 57, no. 3, pp. 3326-3333, 1998.

[7] M. Møller, "Supervised learning on large redundant training sets," Int. J. Neural Syst., vol. 4, no. 1, pp. 15-25, 1993.

[8] G. Maletti, B. Ersbøll, K. Conradsen, and J. Lira, "An initial training set generation scheme," in Proc. 11th Scandinavian Conf. Image Analysis, 2001, pp. 706-714.

[9] J. Lira and G. Maletti, "A supervised contextual classification scheme based on an automated region growing algorithm," in 5th Iberoamerican Symp. Pattern Recognition (SIARP 2000), Lisbon, Portugal, Sept. $11-13,2000$

[10] — , "A supervised contextual classifier based on a region-growth algorithm," Comput. Geosci., vol. 8, no. 8, pp. 951-959, Oct. 2002. 